\title{
“Cool Knees" as a Measure of Systemic Vascular Resistance in Cardiac Patients
}

\author{
Sarah Westcott $^{1}$, William Wung ${ }^{1}$, Aaron Schelegle ${ }^{1}$, Svetlana Ganaga ${ }^{2}$, Saul Schaefer ${ }^{1}$ \\ 1. Internal Medicine, University of California Davis Health System, Sacramento, USA 2. Cardiothoracic Surgery, \\ University of California Davis Health System, Sacramento, USA
}

Corresponding author: Sarah Westcott, skwestcott@ucdavis.edu

\begin{abstract}
Introduction: Clinical assessment of cardiac output (CO) and systemic vascular resistance (SVR) in cardiac patients is often inaccurate. Since the genicular arteries form a watershed zone accessible to physical examination, we hypothesized that "cool knees" would reflect abnormalities in central hemodynamics.
\end{abstract}

Methods: Nineteen patients with cardiac diagnoses, but without distributive shock, had a measurement of skin temperature over the thigh, knee, and foot in parallel with central hemodynamics derived from invasive monitoring.

Results: The temperature gradient from thigh to knee (DTK) reflected increased SVR, and was significantly correlated with SVR, cardiac index (CI), and CO. Cool feet (DTF) were significantly correlated only with systemic hypotension, but not central hemodynamics.

Conclusion: Cool knees reflect increased SVR in cardiac patients and may be an important physical exam finding in their assessment and management.

Categories: Cardiology

Keywords: heart failure, physical examination, systemic vascular resistance, cardiac output, shock

\section{Introduction}

Assessment of perfusion in critically ill patients without sepsis is difficult despite serving a crucial role in the intensive care unit (ICU) management [1]. Studies show that clinicians are highly inaccurate at estimating cardiac output (CO) using physical examination, with some accuracy rates as low as $50 \%[2,3]$. Currently, physicians utilize clinical signs such as capillary refill, skin mottling, heart rate, central venous pressure, and skin temperature to estimate hemodynamic status $[2,4,5]$. Patients are often classified as "cool" or warm" based on the examination of their feet or first toe, leading to a diagnosis of hypoperfusion $[6,7]$. However, feet are thermal regulators and are sensitive to changes in ambient temperature [8]. In contrast, genicular arteries, which provide circulation to the knees, are not thermal regulators and potentially serve as a watershed zone. Thus, their flow would theoretically drop in response to lower systemic perfusion [9], and hence, knee temperature may serve as a more accurate indicator of changes in perfusion. In this study, our aim was to determine if the gradient between thigh skin temperature and knee temperature (DTK; cool knees) would reflect decreased systemic perfusion, and conversely, that cool feet, represented as the gradient between thigh skin temperature and foot skin temperature (DTF) would not reflect changes in perfusion. To the best of our knowledge, this is the first study to investigate the utility of knee temperature measurements in cardiac patients without distributive shock.

\section{Materials And Methods}

All measurements were done following informed consent by the patient or durable power of attorney (DPOA) according to the Helsinki convention following an IRB approved protocol. Our study consisted of 19 patients undergoing hemodynamic monitoring for a variety of cardiac conditions (Table 1) who had simultaneous measurements of vital signs, central hemodynamics acquired from a PA Catheter (Edwards Lifesciences Crop, Irvine, USA), recording of cardioactive medications, and lower extremity temperatures. Skin temperature was measured using an Etekcity Lasergrip 774 Digital Laser Infrared Thermometer (Etekcity Corporation, Anaheim, USA) at the following anatomic points on both lower extremities; knee: over the central portion over the patella, thigh: $10 \mathrm{~cm}$ above the central knee measurement point, and foot: central portion of the dorsum of the foot. Patients were excluded if they had evidence of distributive shock (e.g., sepsis) manifested by elevated central temperature, positive blood cultures, and other signs of system infection. Patients were also excluded if they had a diagnosis of significant peripheral artery disease by abnormal ankle-brachial index, history of claudication, prior imaging evidence, or surgical or endovascular treatment of peripheral arterial disease (PAD). Measurements of lower extremity temperatures were recorded from one lower extremity in instances of amputation, a significant peripheral vascular disease affecting one leg, or a catheter (such as an intra-aortic balloon pump) in the femoral artery. Patient 


\section{Cureus}

characteristics are shown in Table 1.

\begin{tabular}{|c|c|}
\hline Age, mean (SD) & $64.8(15.9)$ \\
\hline Male, n (\%) & $16(84.2)$ \\
\hline White, n (\%) & 15 (78.9) \\
\hline BMI (SD) & $27.7(6.4)$ \\
\hline \multicolumn{2}{|l|}{ Primary diagnosis } \\
\hline Cardiogenic shock, n (\%) & $8(42.1)$ \\
\hline Congestive heart failure exacerbation, $\mathrm{n}(\%)$ & $6(31.6)$ \\
\hline Right heart catheterization, n (\%) & 2 (10.5) \\
\hline Other, n (\%) & $3(15.8)$ \\
\hline History of CAD, n (\%) & $3(15.8)$ \\
\hline History of diabetes mellitus, n (\%) & $4(21.1)$ \\
\hline Vasopressors, n (\%) & $6(31.6)$ \\
\hline
\end{tabular}

TABLE 1: Baseline demographics for patients with recorded thigh to knee temperature measurements, $n=19$.

BMI: body mass index, CAD: coronary artery disease.

Data were characterized as mean \pm standard deviation for continuous variables and as number and percentages for categorical variables. Correlation coefficients were calculated using logistic regression and are expressed as R-values and significance F-values. Statistical significance was at $\mathrm{p}<0.05$. All statistical analysis was performed with R version 3.5.1 (R Foundation for Statistical Computing, Vienna, Austria).

\section{Results}

In support of the hypothesis, there was a significant relationship between DTK and SVR as well as CO and cardiac index (CI; Figure 1).

$1 \mathrm{~A}$
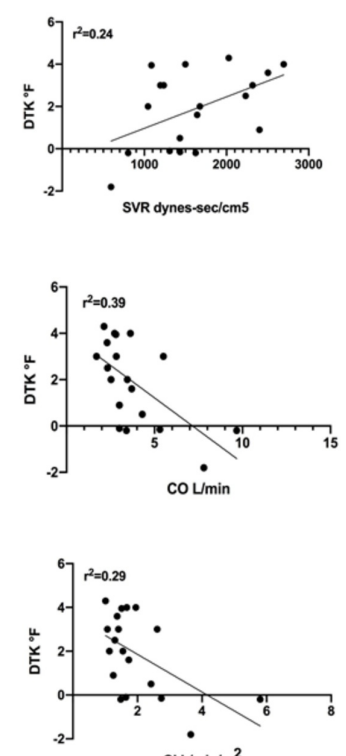


\section{Cureus}

\section{FIGURE 1: (A) SVR, (B) CO, and (C) Cl versus change in temperature}

from thigh to knee (DTK).

SVR: systemic vascular resistance, CO: cardiac output, Cl: cardiac index.

Specifically, DTK increased as SVR increased. In terms of clinical utility, of the 10 patients with an SVR > 1500 dynes-sec $/ \mathrm{cm}^{5}$, only one had "warm knees," i.e., DTK < $1 \mathrm{oF}$, while $8 / 11$ patients with a DTK > $1 \mathrm{oF}$ had an SVR > 1500 dynes-sec $/ \mathrm{cm}^{5}$. While CI and CO were significantly correlated to DTK, a larger DTK did not reliably identify those patients with lower $\mathrm{CI}$ and $\mathrm{CO}$ as many patients had reduced values. There was no significant relationship between DTK and other hemodynamics measurements, including systolic blood pressure (SBP) and pulmonary capillary wedge pressure (PCWP; Table 2).

\begin{tabular}{|c|c|c|}
\hline & R-value & Significance $\mathbf{F}$ \\
\hline \multicolumn{3}{|c|}{$\Delta$ Thigh to knee temperature (DTK) } \\
\hline SVR & 0.58 & 0.01 \\
\hline $\mathrm{CO}$ & 0.62 & 0.006 \\
\hline $\mathrm{Cl}$ & 0.53 & 0.02 \\
\hline SBP & 0.21 & 0.47 \\
\hline PCWP & 0.11 & 0.63 \\
\hline \multicolumn{3}{|c|}{$\Delta$ Thigh to foot temperature (DTF) } \\
\hline SVR & 0.05 & 0.84 \\
\hline $\mathrm{CO}$ & 0.38 & 0.13 \\
\hline $\mathrm{Cl}$ & 0.36 & 0.16 \\
\hline SBP & 0.61 & 0.02 \\
\hline PCWP & 0.41 & 0.12 \\
\hline
\end{tabular}

\section{TABLE 2: Regression analysis results of catheterization results versus change in temperature} from thigh to knee (DTK) and thigh to foot (DTF).

SVR: systemic vascular resistance, CO: cardiac output, $\mathrm{Cl}$ : cardiac index, SBP: systolic blood pressure, PCWP: pulmonary capillary wedge pressure.

In contrast to DTK, DTF was not significantly related to SVR, CO, or CI $(\mathrm{R}=0.05,0.38$, and 0.36 , respectively). However, a higher DTF (i.e., “cool feet”) was significantly correlated with systemic blood pressure $(\mathrm{R}=0.61)$, with the temperature gradient increasing with lower blood pressure.

\section{Discussion}

These findings support the use of the temperature gradients from the thigh to knee (DTK) as an assessment of systemic hypoperfusion and elevated SVR in patients without distributive shock. The temperature gradient from the thigh to foot (DTF) was not helpful in assessing these central hemodynamic variables, although cooler feet did reflect systemic hypotension. The current study is unique in that it only included patients with cardiac diagnoses and used skin temperature measurements with a laser infrared thermometer that can be commercially obtained for less than $\$ 30$ (e.g., Etekcity Lasergrip 774 Digital Laser Infrared Thermometer). Other studies have concluded that extremity temperatures are a weak indicator of perfusion status, probably due to the inclusion of a broad category of patients (many with distributive shock) and the use of clinical assessments such as knee mottling and "cool extremities" rather than objective knee temperature measurements $[2,6,10]$. In addition to the DTK serving as a real-time assessment of SVR in cardiac patients, measurement of DTK could potentially assess changes in perfusion over time in individual patients. Further studies are needed to investigate this relationship as well as the sensitivity/specificity of these clinical exam findings. 
Due to the declining use of pulmonary artery catheters to manage critically ill patients, a limited number of patients who were largely Caucasian and male could be enrolled in the study period. Thus, the statistical power and clinical import of these findings is limited and should be confirmed with a larger, more diverse, set of patients. In addition, there was no threshold value of DTK that identified patients with a reduced CI or $\mathrm{CO}$ indicative of cardiogenic shock. This can be attributed to the limited sample size, the fact that most patients had a CI below $2.2 \mathrm{~L} / \mathrm{min} / \mathrm{m}^{2}$, as well as interventions that were instituted as part of clinical care.

\section{Conclusions}

“Cool knees," defined as an increase in DTK, represents systemic hypoperfusion via elevated SVR. DTK may serve as an additional vital sign that can be used to help manage critically ill patients with cardiac disease in combination with other validated clinical exam findings. In contrast, while "cool feet" reflect hypotension in cardiac patients, they do not reflect systemic hemodynamics and should not be used to assess perfusion in these patients.

\section{Additional Information \\ Disclosures}

Human subjects: Consent was obtained by all participants in this study. University of California Davis issued approval 975039-4. This study protocol was approved by the Institutional Review Board of the University of California Davis. Animal subjects: All authors have confirmed that this study did not involve animal subjects or tissue. Conflicts of interest: In compliance with the ICMJE uniform disclosure form, all authors declare the following: Payment/services info: All authors have declared that no financial support was received from any organization for the submitted work. Financial relationships: All authors have declared that they have no financial relationships at present or within the previous three years with any organizations that might have an interest in the submitted work. Other relationships: All authors have declared that there are no other relationships or activities that could appear to have influenced the submitted work.

\section{References}

1. Cecconi M, De Backer D, Antonelli M, et al.: Consensus on circulatory shock and hemodynamic monitoring. Task force of the European Society of Intensive Care Medicine. Intensive Care Med. 2014, 40:1795-1815. 10.1007/s00134-014-3525-Z

2. Hiemstra B, Eck RJ, Keus F, van der Horst IC: Clinical examination for diagnosing circulatory shock. Curr Opin Crit Care. 2017, 23:293-301. 10.1097/MCC.0000000000000420

3. Hiemstra B, Koster G, Wiersema R, et al.: The diagnostic accuracy of clinical examination for estimating cardiac index in critically ill patients the simple intensive care studies - I. Intensive Care Med. 2019, 45:190200. 10.1007/s00134-019-05527-y

4. Ferraris A, Bouisse C, Mottard N, Thiollière F, Anselin S, Piriou V, Allaouchiche B: Mottling score and skin temperature in septic shock: relation and impact on prognosis in ICU. PLoS One. 2018, 13:e0202329. 10.1371/journal.pone.0202329

5. Vazquez R, Gheorghe C, Kaufman D, Manthous CA: Accuracy of bedside physical examination in distinguishing categories of shock: a pilot study. J Hosp Med. 2010, 5:471-474. 10.1002/jhm.695

6. Kaplan LJ, McPartland K, Santora TA, Trooskin SZ: Start with a subjective assessment of skin temperature to identify hypoperfusion in intensive care unit patients. J Trauma. 2001, 50:627-628. 10.1097/00005373200104000-00005

7. Henning RJ, Wiener F, Valdes S, Weil MH: Measurement of toe temperature for assessing the severity of acute circulatory failure. Surg Gynecol Obstet. 1979, 149:1-7. http://www.ncbi.nlm.nih.gov/pubmed/451819

8. Taylor NA, Machado-Moreira CA, van den Heuvel AM, Caldwell JN: Hands and feet: physiological insulators, radiators and evaporators. Eur J Appl Physiol. 2014, 114:2037-2060. 10.1007/s00421-014-2940-8

9. Shahid S, Saghir N, Cawley O, Saujani S: A cadaveric study of the branching pattern and diameter of the genicular arteries: a focus on the middle genicular artery. J Knee Surg. 2015, 28:417-424. 10.1055/s-00351549021

10. Schey BM, Williams DY, Bucknall T: Skin temperature and core-peripheral temperature gradient as markers of hemodynamic status in critically ill patients: a review. Heart Lung. 2010, 39:27-40. 10.1016/j.hrtlng.2009.04.002 